\title{
Holography from Venus de Milo to cultural performance, science and technology (Withdrawal Notice)
}

Patrice Salzenstein

Patrice Salzenstein, "Holography from Venus de Milo to cultural performance, science and technology (Withdrawal Notice)," Proc. SPIE 10233, Holography: Advances and Modern Trends V, 102331N (15 May 2017); doi: $10.1117 / 12.2264895$

SPIE. Event: SPIE Optics + Optoelectronics, 2017, Prague, Czech Republic 


\section{Holography from Venus de Milo to cultural performance, science and technology (Withdrawal Notice)}

Patrice Salzenstein

CNRS, Univ. de Bourgogne Franche-Comté, FEMTO-ST Institute (France)

Proc. SPIE 10233, 102331N (2017)

Online Publication Date: 15 May 2017

Withdrawn from Publication: 22 May 2017

Conference Date: 24-27 April 2017

Conference Location: Prague, Czech Republic

Conference Title: Holography: Advances and Modern Trends

Conference Chairs: Miroslav Hrabovský, John T. Sheridan, Antonio Fimia

This paper has been withdrawn by the publisher at the request of the author. 